\title{
Improving the Performance of MANET Gateway Selection Scheme for Disaster Recovery
}

\author{
Nor Aida Mahiddin \\ School of Engineering, Computer and Mathematical \\ Sciences, Auckland University of Technology \\ Auckland, New Zealand \\ nmahiddi@aut.ac.nz
}

\begin{abstract}
In this paper, we propose an improved MANET gateway selection scheme suitable for disaster recovery applications. Having an infrastructure less and decentralize features, MANET is well suited to bring the network back that has been collapse after a disaster. We focus on improving throughput performance of MANET by designing a better gateway selection scheme. The key idea is to eliminate the congestion at each MANET gateway for improved performance. Simulation results show that the proposed gateway selection scheme can efficiently manage the traffic distribution at each gateway to maximize the network performance.
\end{abstract}

Keywords-MANET; Gateway Selection Scheme; Disaster Recovery Area; Throughput.

\section{INTRODUCTION}

A MANET is a group of mobile nodes (MNs) which the communications are performed through multi-hop routing using the multi-hop wireless link. By forwarding packets to the neighbors, each node plays an important role not only as a user but also as a relay. The advantage of MANET is network can be performing without any support from existing infrastructure. It can rapidly form and deform a network without decentralized management. Therefore, MANET is suitable to be one of the solutions for communication after a disaster occurs.

In post-disaster areas, collapse building, communication infrastructures damaged and become unworkable, are a common result. We know that natural disasters (e.g., earthquake, tsunami, typhoon and etc.) occur frequently over the years in the worldwide, which cause the destruction of a large number communication equipment's (e.g., base station, wireless router). It is extremely expensive and time-consuming to replace or repair if major installations such as cell towers or fiber optic cables are involved. Thus, to keep communication alive in a disaster recovery area, MANET features and advantages are very feasible for disaster recovery area. However, victims seek to contact family and friends cause to a high level of data traffic which leads to network congestion.

To allow communication between MANET nodes and the outside network, it requires a gateway as a door to let entry and exit packets from the network. This gateway is the Internet Gateway (IG) which will route all packets to and from the Internet. A gateway is also a node in MANET networks. The

\author{
Nurul I Sarkar \\ School of Engineering, Computer and Mathematical \\ Sciences, Auckland University of Technology \\ Auckland, New Zealand \\ nurul.sarkar@aut.ac.nz
}

main task of a gateway is to control network traffic between two or more different networks. In one network it can have more than one gateway. As shown in Fig. 1. each gateway has an average queue size to monitor. On the other hand, congestion may occur when the number of packets being transmitted to the gateway exceeded pre-set threshold queue size. Therefore, how MNs choose a gateway has been a key issue in recent years as it affects the network throughput performance.

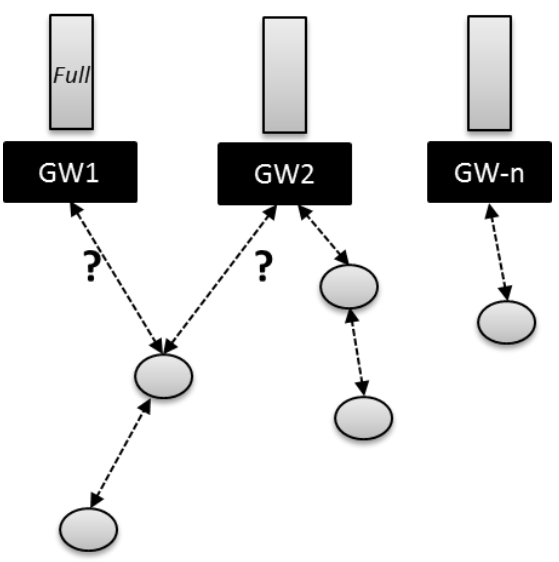

Fig. 1. Gateway selection scheme plays an important role to achieve better network performance.

In traditional wireless network algorithm, MNs will choose the nearest gateway to send data packet regardless of the heavy traffic load[1]. The bottleneck queuing at gateway leads to congestion and packet loss [2]. Imbalance traffic distribution among MANET gateways causes network performance degradation. Therefore, in this paper, we would like to introduce an enhancement of gateway selection scheme in disaster recovery areas to maintain the throughput performance in MANET. The main contribution of this research is to optimize the throughput for MANET performances by developing a gateway selection schemes with consideration of nodes mobility.

The remainder of this paper is organized as follows: In section II we introduce briefly about MANET gateway on previous research and the challenge of nodes mobility. In section III, we discuss in details a gateway selection method. 
While simulation and performance evaluation are devoted in section IV. Finally, we conclude the paper in section V.

\section{RELATED WORK}

\section{A. MANET Gateway / Gateway throughput}

Recently, there are many studies about Gateway. As MANET itself, gateways are the entry points for nodes in MANET to connect to the external network. There might be a scenario where after a disaster occurs, communication network fail and it took a period of time to restore the communication that has been damaged. Therefore, since MANET characteristics can be an immediate solution for this scenario, the main challenges is heavy traffic load as people want to contact their family and friends.

Therefore, in this section, we will discuss the previous study of gateway selection scheme. It has been observed that some of the proposed technique are actually modifying from the traditional routing protocol method like AODV and DSDV [3] [4] [5]. In the research conducted by Tashtoush et al. [6], proposed a method by using hop count as a weight value to the gateway. To choose the effective route, it limits the number of routes via weight of Fibonacci. The smallest hop count will be selected as a route to the gateway. However, the keep running computation of route weight has affected to network overhead.

In addition, to recover communications in a disaster area, Liu et al. [7] present a solution to straightforwardly find gateway nominees without heavy computation load. Nonetheless, this method considers one single channel and only one gateway will serve in one area. Because of throughput is one of the criteria for performance evaluation, by electing different gateway will change throughput in network performance.

Furthermore, Prabhavat et al. [8] has evaluated many load distribution method over multipath network with different criteria. It clearly mentions the main role to achieve better performance for load balancing in the network is the technique of splitting the traffic and route selection. However, this study does not discover routing method to establish multiple paths. Load balancing scheme is a part of how gateway being selected and how each node chooses the particular gateway for packet transmission. Congestion may occur as many nodes focus on the same gateway. Hence, the efficient techniques to maintain packets ordering with the purpose of preventing packet loss are very important as an efficient technique can maximize the throughput performance.

\section{B. Mobility nodes}

The mobility of nodes in MANET has significantly affected the network performances. Mobility leads to frequent topology change and these are challenges task to design an efficient gateway selection scheme. As topology rapidly changes due to node movements in Ad Hoc networks, this situation may head to packet losses or delay. Transmission Control Protocol (TCP) is a Transport Protocol primarily for reliable, ordered and error checked delivery. TCP has misinterpreted status of the route as congestion and appeals congestion control. Chandran et al. [9] introduced a Feedback scheme to overcome route failure during packet transmission from sender to receiver. When the route changes, the node sends Route Failure Notification (RFN) packet to the sender to freeze its timer and stop sending the next packets. When the route has re-established, packet Route Re-establishment Notification (RRN) are sent to resume timers and continuing packet transmission. Thus, packet retransmissions are required and this may lead to delay and unfairness of packet throughput.

Case studies in the pastoral area of Tibet have been used to support mobile communication efficiently. To allow people interconnect through the Internet, [10] proposed wireless networking architecture to connect MANET to Cellular network via a Terrestrial gateway and then Cellular network will connect between the MANET and Internet. L. Mu et al. [11] used almost the same case study as Huang et al. proposed, which is involved Communication Architecture for Maritime Sectors which is using the integration of Cellular, Satellite, WiMax, and Wi-Fi. While access to Cellular coverage is limited, [12] introduced a combination of MANET and cellular to achieve enhancement of delivery packet ratio in mobile ad hoc network.

In mobility node, before communication started, each node will broadcast information of its coordinate and current moving speed to other nodes within maximum transmission range of each node. Then, each node assembles the information of their neighbors and builds its own graph. Each node has owned moving speeds. Nishima et al. [13] introduced a dynamic method to measure performance metric in MANET in terms of connectivity ratio. $\mathrm{Li}$ et al. proposed topology control algorithm namely Local Minimum Spanning Tree (LMST) and [14] proposed Local Treebased Reliable Topology (LTRT), which is the combination idea of LMST and TRT. LMST is the most cost effective because each node only has one path. There is no path redundancy. But the biggest problem is when one of the link failures, it will be no connectivity to the related node. Hence, [14] introduced LTRT algorithm, a mathematical solution to assurance k-edge connectivity of the topology. On the other hand, [15] measure the error of hop count based on distance approximation and classifies the main influencing factors is mobility. There are some factors taken into account that affect the mobility in mobile ad hoc network such as speed, direction, and similarity of moves in neighborhoods. Movement of devices in MANET highly depends on the application and the environment.

To simulate and evaluating the performance of a new scheme, identifying mobility model plays an important role in describing the movements pattern of the mobile nodes in MANETs environment. There are seven different models for node mobility. However, only two of them are commonly used. The first model is Random walk mobility and the second model is Random waypoint mobility. Random waypoint mobility model [16] is usually used in MANET routing scheme because of its easiness and wide availability. According to the behavior of this model, before node changing the direction or speed, it will include pause times. In advances before time duration 
expires, the node will randomly choose next destination within range and speed. After node complete moves to selected destination, it has again had to wait after pre-set pause time before the same process can be repeated. Figure 5 shows an example of a mobile node that uses the random waypoint mobility model as a traveling pattern. The movement pattern of this model is similar to Random walk mobility model if pause time is zero. In most of the research mobile ad hoc performance evaluation, this model is commonly used in a simulation.

\section{A GATEWAY SELECTION METHOD}

To keep communications alive, MANET characteristics are significantly helpful to be deployed in post-disaster. Fig.1. shows an example of MANET infrastructure in disaster recovery area. Here, we configure some nodes in MANET to be a gateway. This gateway will be a relay for others node in MANET to the Internet. These gateways are chosen because the nodes are in wireless access point coverage. If a node in MANET wants to send a packet to Internet, and that node is not in a gateway range, the neighbor's node will forward the packet to the upper-level route using our proposed routing selection scheme as in our previous paper [17] until it arrives at the gateway. In this paper, we assume electricity and power are not damage or having a backup. We also concern about the security of the nodes, however, it is outside our paper research scope.

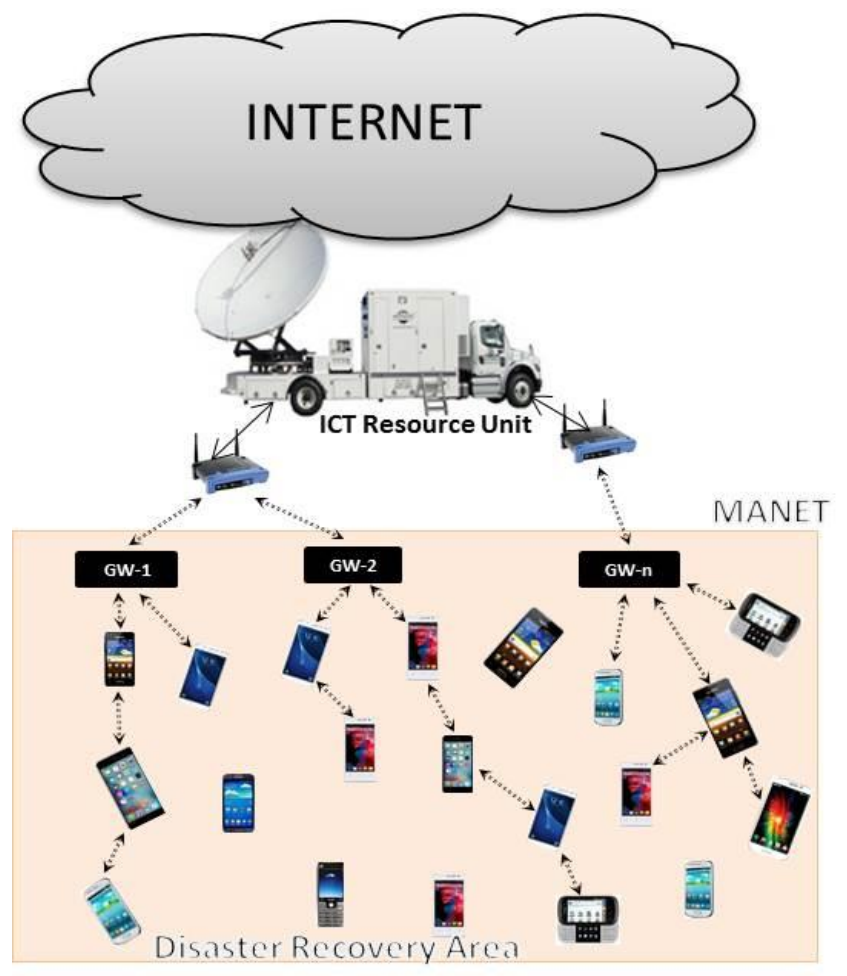

\begin{tabular}{|l|l|l|l|}
\hline$\longleftrightarrow$ & Wired Link & $\leftrightarrow \cdots \cdots \cdots \cdots \cdots$ & Wireless Link \\
\hline
\end{tabular}

Fig. 2. An example MANET infrastructure in disaster recovery area.
In a disaster recovery area, when we set up a MANET to recover communications, certainly cannot be avoided the heavy load of data traffic as victims seek to contact family and friends. Nodes in MANET send a packet to the nearest gateway regardless of the gateway load. The bottleneck at one gateway occurs when many nodes send a packet to the same gateway at the same time. As a consequence, to overcome the issue of heavy load traffic at one gateway and the others are light, we proposed a gateway selection scheme to manage the load balancing between gateways, for optimize the throughput of MANET performance. The scheme as follows:

Step 1: In our scheme, we have proposed technique where only neighbors of the gateway (nodes within gateway range) will receive an advertisement and notification. This technique is to prevent numbers of packet flooding in the network. Therefore, the selected nodes that receive the advertisement will store the information of nearest gateway.

Step 2: Furthermore, if neighbor's received a notification of heavy load from one of the gateways, the particular gateway is no longer be able to receive any other packet in a certain time. Thus, the node will find others gateway in their range to send the packet. The notification of heavy load from gateway can effectively reduce the number of packet loss as to eliminate the congestion in MANET gateways.

Step 3: On the other hand, if the gateway is out of the range, the node will send the packet to the upper-level node within node range (base on our routing selection scheme). Neighbor's node in MANET will forward the packet until the packet reaches to the gateway. This method has significantly reduced the complexity of routing selection scheme consequently improved packet delay.

Step 4: Additionally, we consider the mobility of nodes since nodes in MANET move randomly and they can connect each other wirelessly. Because of nodes are mobile, the mobility has a significant impact on the MANET performance.

Step 5: Finally, we determine MANET performance of our proposed scheme through the observation of packet loss, packet delay and throughput at each gateway.

\section{PERformance Evaluation}

In this section, we conducted our proposed scheme using a network simulation tools. To evaluate the performance, we analyze the results obtained from each gateway. The results are presented following the explanation of our simulation setup.

\section{A. Simulation setup}

We simulate an environment of disaster recovery area in OMNET++ simulation tools within $1200 \mathrm{~m} \times 800 \mathrm{~m}$ with 100 nodes distributed in the area. The transmission range of each node is equal to $250 \mathrm{~m}$. Using a Random Waypoint model, the mobility speeds are set to $2 \mathrm{mps}$ and the data rate is $2 \mathrm{Mbps}$. Simulation time is fixed to 900 (s). In this simulation, among 100 nodes, we configured node 8,15 , and 49 to be a gateway. We assume these three nodes had received Wireless Internet coverage. Hence, these nodes will be a gateway to all MANET nodes that do not receive Internet coverage. The destination of 
all $\mathrm{MN}$ in disaster recovery area is a gateway. Beginning with gateways initialized current positions and then determining who gateway's neighbor is, followed by nodes at each level determining their neighbor to discover the shortest route to the gateway. All the simulation environment used are summarized in Table 1.

TABLE I. SIMULATION ENVIRONMENT

\begin{tabular}{|l|l|}
\hline Parameter & \multicolumn{1}{|c|}{ Value } \\
\hline Simulation Area $\left(\mathrm{m}^{2)}\right.$ & $1200 \times 800$ \\
\hline Simulation Time (s) & 900 \\
\hline Mobility Model & Random Waypoint \\
\hline Mobile Node Placement & Random \\
\hline Pause Time (s) & $0-2$ \\
\hline Transmission Range (m) & 250 \\
\hline Packet Size & 512 bytes \\
\hline Traffic Type & CBR \\
\hline Wireless MAC Interface & IEEE $802.11 \mathrm{~b}$ \\
\hline Number of Gateway & 3 \\
\hline Node Speed (m/s) & 2 \\
\hline Number of Mobile Nodes & 100 \\
\hline
\end{tabular}

\section{B. Comparing throughput between each gateway}

By using the simulation result, we observed the load balance deviation between each gateway to measure the packet throughput in MANET performance. In the evaluation, our proposed scheme result show respective throughput of Gateway 1, Gateway 2 and Gateway 3. Fig. 3. illustration show nearly perfect load balancing, since the throughput of these three gateways almost equal until the number of nodes increases to hundred. The largest throughput we can find at Gateway2 when a number of nodes are 50. However, the throughput decreases slowly as the number of nodes increases.

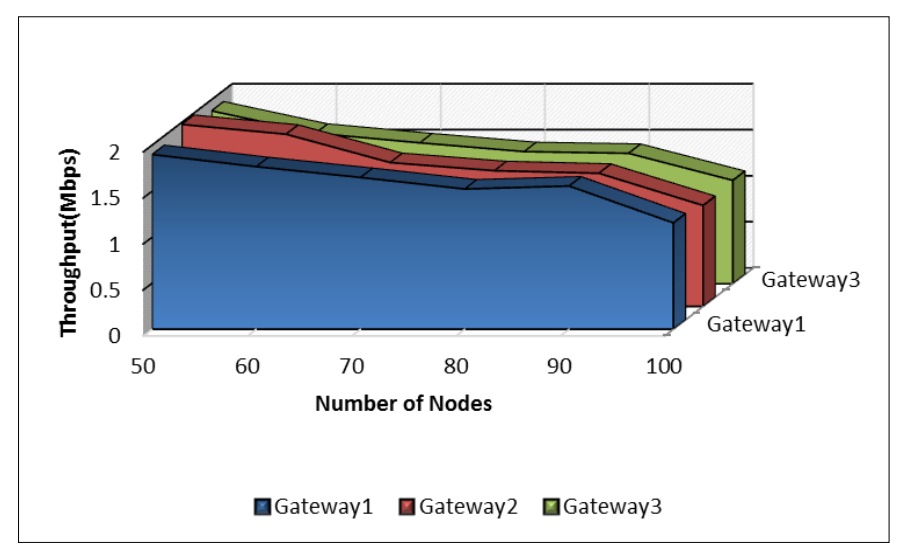

Fig. 3. Comparison of the throughput to determine load balance between gateways.
The different distributed traffic loads at gateway will affect bottleneck which leads to packet loss and packet delay. From Fig. 4. we can see there is zero packet loss up to a number of nodes 60 . In other words, with our proposed gateway selection scheme, imbalance problem at each gateway can be solved therefore packet drop ratio can be reduced. Only when a number of nodes are growth to 70, packet loss slightly increase. Nevertheless, traffic distribution of our scheme still succeeds to avoid traffic congestion.

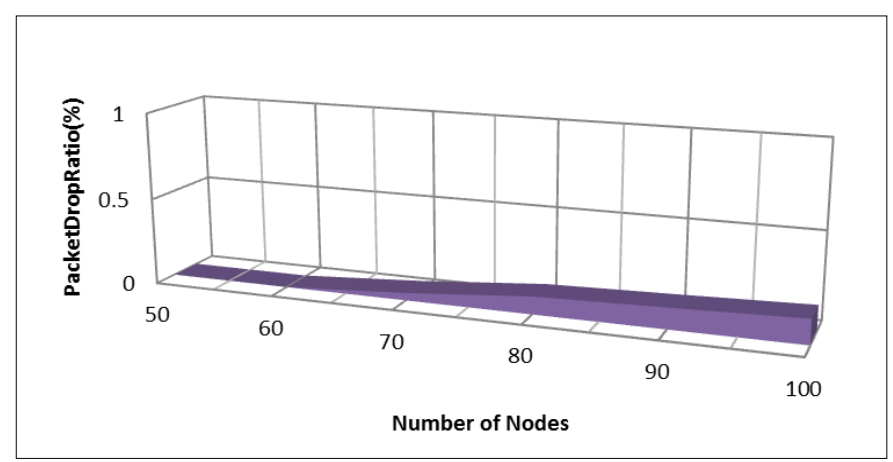

Fig. 4. The ratio of packet drops in MANET

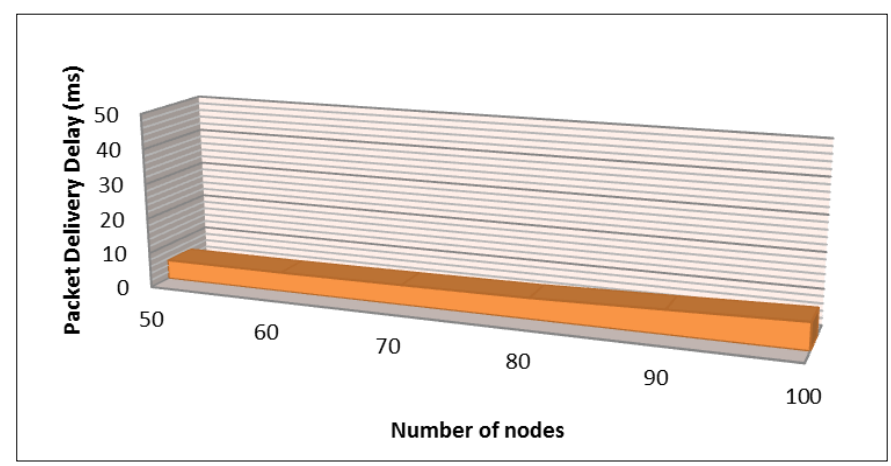

Fig. 5. Packet Delivery Delay for the number of nodes.

Furthermore, as shown in Fig. 5 we can confirm that our proposed gateway selection scheme achieves a lower packet delivery delay as the number of nodes increase, thus the packet delivery delay was still small. Therefore, overall MANET performance of this simulation results shows the effectiveness of our scheme. The result of the scheme revealed that we have made an improvement in MANET performance through gateway selection scheme. Total throughput of the whole network, small packet loss, and packet delay, clearly enhancing MANET performance. On the other hand, each user in disaster recovery area can still send messages even with higher traffic load. In addition, the results of this simulation can efficiently deliver Internet connectivity to the people in the disaster area with a significantly high number of the user. 


\section{CONCLUSION}

In this paper, we proposed a gateway selection scheme to improve MANET throughput in disaster recovery areas. Simulation results have shown that the proposed gateway selection scheme can provide improved network performance. The proposed gateway selection method can be used in other networks, such as wireless mesh network and Internet of Things for improved performance.

\section{REFERENCES}

[1] A. Mahiddin, R. Hasyifah, W. Norshuhadah, and W. Nik, "Performance Comparison of Packet Queuing Algorithm in Wireless Networks," Int. J. Inf. Technol. Comput. Sci., vol. 13, no. 2, pp. 18-24, 2014.

[2] N. A. Mahiddin, A. Nazari, B. Mohd, and S. Subramaniam, "An Intelligent Buffer Management For Packet Scheduling Algorithm in Multihop Wireless LANs," Int. J. Futur. Gener. Commun. Netw., vol. 5, no. 2, pp. 97-106, 2012.

[3] Y. Miao, Z. Sun, F. Yao, N. Wang, and H. S. Cruickshank, "Study on Research Challenges and Optimization for Internetworking of Hybrid MANET," in Personal Satellite Services, Springer, 2013, pp. 90-101.

[4] R.-U. Zaman, K.-U.-R. Khan, and a. V. Reddy, "A review of gateway load balancing strategies in Integrated InternetMANET," 2009 IEEE Int. Conf. Internet Multimed. Serv. Archit. Appl., pp. 1-6, Dec. 2009.

[5] Q. Le-trung, P. E. Engelstad, T. Skeie, and A. Taherkordi, "LoadBalance of Intra / Inter-MANET Traffic over Multiple Internet Gateways," in MoMM '08 Proceedings of the 6th International Conference on Advances in Mobile Computing and Multimedia, 2008, no. 0316, pp. 50-57.

[6] Y. Tashtoush, O. Darwish, and M. Hayajneh, "Fibonacci sequence based multipath load balancing approach for mobile ad hoc networks," Ad Hoc Networks, vol. 16, pp. 237-246, 2014.

[7] W. Liu, H. Nishiyama, N. Kato, Y. Shimizu, and T. Kumagai, “A novel gateway selection method to maximize the system throughput of Wireless Mesh Network deployed in disaster areas," IEEE Int. Symp. Pers. Indoor Mob. Radio Commun. PIMRC, no. Pimrc, pp. 771-776, 2012.

[8] S. Prabhavat, H. Nishiyama, and N. Ansari, "On Load Distribution over Multipath Networks," IEEE Commun. Surv. Tutorials, vol. 14, no. 3, pp. 662-680, 2012.

[9] K. Chandran, S. Raghunathan, S. Venkatesan, and R. Prakash, "A Feedback Based Scheme For Improving TCP Performance In Sudarshan Raghunathan," IEEE Personal Communication Magazine, Special Issue on Ad Hoc Networks, pp. 34-39, 2001.

[10] C. Huang, X. Guo, and Z. Liu, "A wireless networking architecture using MANET for mobile communications of remote pastoral areas in Tibet," in 2nd International Conference on Computer Science and Electronics Engineering (ICCSEE 2013), 2013, no. Iccsee, pp. 800-803.

[11] L. Mu, R. Kumar, and A. Prinz, "An Integrated Wireless Communication Architecture for Maritime Sector," LNCS, Springer, pp. 193-205, 2011.

[12] B. Bhargava, X. Wu, Y. I. Lu, and W. Wang, "Integrating Heterogeneous Wireless Technologies : A Cellular Aided Mobile Ad Hoc Network ( CAMA )," in Mobile Networks and Applications, Kluwer Academic Publishers, 2004, pp. 393-408.

[13] H. Nishiyama, T. Ngo, N. Ansari, and N. Kato, "On Minimizing the Impact of Mobility on Topology Control in Mobile Ad Hoc Networks," IEEE Trans. Wirel. Commun., vol. 11, no. 3, pp. 1158-1166, 2012.

[14] K. Miyao, H. Nakayama, N. Ansari, and N. Kato, "LTRT: An Ef fi cient and Reliable Topology Control Algorithm for Ad-Hoc Networks," IEEE Trans. Wirel. Commun., vol. 8, no. 12, pp. 6050-6058, 2009.

[15] S. Merkel, S. Mostaghim, and H. Schmeck, "Hop count based distance estimation in mobile ad hoc networks - Challenges and consequences," Ad Hoc Networks, vol. 15, pp. 39-52, Apr. 2014.
[16] S. K. Sarkar, T. G. Basavaraju, and C. Puttamadappa, Ad Hoc Mobile Wireless Networks: Principles, Protocols and Applications, 2nd Editio. Taylor \& Francis Group, 2013.

[17] N. A. Mahiddin, N. I. Sarkar, and Brian Cusack, "Gateway Load Balancing and Routing Selection Scheme of MANET in Disaster Scenario," in 2015 2nd Asia-Pasific World Congress on Computer Science and Engineeering (APWC on CSE), 2015, vol. 1542 , pp. 1-7. 